STRUCTURAL BIOLOGY COMMUNICATIONS

ISSN 2053-230X

Received 29 December 2016

Accepted 27 March 2017

Edited by W. N. Hunter, University of Dundee, Scotland

Keywords: d(CCGGGGTACCCCGG) 2 ; A-DNA duplex; X-ray crystallography; tetragonal space group.

PDB reference: d(CCGGGGTACCCCGG $)_{2}$, $5 \mathrm{wv} 7$

Supporting information: this article has supporting information at journals.iucr.org/f

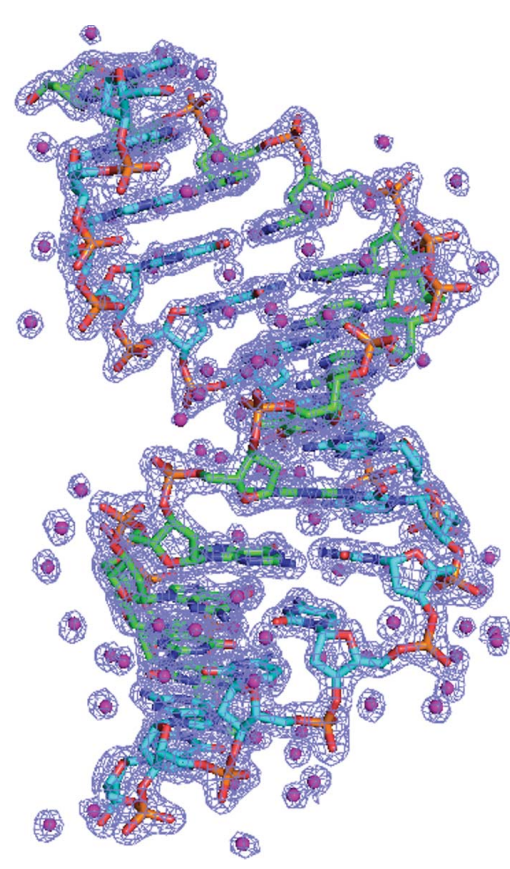

C 2017 International Union of Crystallography

\section{Crystal structure of $\mathrm{d}\left(\mathrm{CCGGGGTACCCCGG)_{2 }}\right.$ at $1.4 \AA$ resolution}

\author{
Selvam Karthik, ${ }^{a}$ Arunachalam Thirugnanasambandam, ${ }^{a}$ Pradeep Kumar Mandal ${ }^{b}$ \\ and Namasivayam Gautham ${ }^{\text {a* }}$
}

${ }^{a}$ CAS in Crystallography and Biophysics, University of Madras, Guindy Campus, Chennai 600 025, India, and ${ }^{\mathbf{b}}$ Institut Européen de Chimie et Biologie (IECB), 2 Rue Robert Escarpit, 33607 Pessac CEDEX, France. *Correspondence e-mail: n_gautham@hotmail.com

The X-ray crystal structure of the DNA tetradecamer sequence d(CCGGGGT$\mathrm{ACCCCGG})_{2}$ is reported at $1.4 \AA$ resolution in the tetragonal space group $P 4_{1} 2_{1} 2$. The sequence was designed to fold as a four-way junction. However, it forms an A-type double helix in the presence of barium chloride. The metal ion could not be identified in the electron-density map. The crystallographic asymmetric unit consists of one A-type double helix with 12 base pairs per turn, in contrast to 11 base pairs per turn for canonical A-DNA. A large number of solvent molecules have been identified in both the grooves of the duplex and around the backbone phosphate groups.

\section{Introduction}

In 2011, our laboratory reported the first structure of a tetradecadeoxynucleotide, d(CGCGGGTACCCGCG) $)_{2}$, in the A-DNA conformation (Venkadesh et al., 2011). Following this, a series of tetradecamer sequences such as d(CCCCGGTACCGGGG) $)_{2}$ (Mandal et al., 2012; Purushothaman et al., 2014), d(CCCGGGTACCCGGG) ${ }_{2}$ (Karthik et al., 2017) and the present sequence have all been reported to crystallize as A-type double helices. These sequences have some common features. (i) All of them contain the KpnI restriction site GGTACC in the centre, flanked by combinations of purines and pyrimidines. They all have a 'TA' step at the centre. (ii) All of the sequences crystallized in the tetragonal system, either in space group $P 4_{1}$ or $P 4_{1} 2_{1} 2$. (iii) The change in space group between the structures has been attributed to two aspects: different inter-helical interactions and the presence of a well defined manganese ion in $\mathrm{d}(\mathrm{CCCCGGTACCGGGG})_{2}$ and a zinc ion in $\mathrm{d}(\mathrm{CCCGGGTACCCGGG})_{2}$. However, the exact role of the metal ions in bringing about the differences in crystal packing and space group is unknown. (iv) Despite possessing sequence symmetry, the two heptameric halves are structurally different. The helical parameters and inter-helical interactions are not symmetrically distributed in the two halves of the helix. (v) The terminal base pairs of the helix are structurally quite different from the central base pairs. (vi) The most common inter-helical interaction in these A-DNA crystals is the stacking of terminal base pairs over the minor groove of a symmetry-related helix. Other types of interhelical interactions are also present in the crystals.

Here, we present the crystal structure of the tetradecanucleotide d(CCGGGGTACCCCGG) $)_{2}$ refined to $d_{\min }=$ $1.4 \AA$ A. The article describes the major structural features of the 
duplex and its hydration, and elucidates the crystal-packing interactions. A comparison has been made with fibre A-DNA and the average values from high-resolution crystal structures in order to deduce the possible sequence-dependent microheterogeneities.

\section{Materials and methods}

Purified synthetic DNA samples and other chemicals were purchased from Sigma-Aldrich Chemicals Pvt. Ltd, Bengaluru, India. Crystallization trials were conducted using the hanging-drop vapour-diffusion method at $293 \mathrm{~K}$. Good crystals were obtained after $4 \mathrm{~d}$ from a drop consisting of $1 \mathrm{~m} M$ DNA, $50 \mathrm{~m} M$ sodium cacodylate trihydrate buffer $\mathrm{pH}$ 7,1 $\mathrm{m} M$ $\mathrm{BaCl}_{2}, 5 \mathrm{~m} M$ spermine equilibrated against $50 \%(v / v)$ MPD as a reservoir solution. The crystal was mounted on a cryoloop and flash-cooled in liquid nitrogen before being shipped for data collection.

X-ray data collection was carried out on the BM14 beamline at the European Synchrotron Radiation Facility, Grenoble, France at a wavelength of $0.9737 \AA$ using a MAR CCD 225 detector. The data were indexed, integrated and scaled using $X D S$ (Kabsch, 2010). A summary of the data-processing statistics is provided in Table 1 . The reported resolution of $1.4 \AA$, at which the structure was refined, is based on the correlation coefficient $\left(\mathrm{CC}^{*}\right)$ between the data and the model (Supplementary Fig. S1) and paired refinement results (Supplmentary Table S1) (Karplus \& Diederichs, 2012). Calculations using phenix.xtriage (Zwart et al., 2005) showed no significant anomalous signal; therefore, Friedel pairs were treated as symmetry-equivalent reflections. The following tests were also carried out before proceeding to structure solution. (i) The Matthews coefficient (Matthews, 1968) value of $2.12 \AA^{3} \mathrm{Da}^{-1}$ suggested that there is one duplex in the asymmetric unit. (ii) Inspection of the native Patterson (Rossmann et al., 1964) indicated the absence of noncrystallographic symmetry. (iii) The $L$-test for acentric reflections (Padilla \& Yeates, 2003) suggested that the data were not twinned. (iv) The data set was reprocessed in the triclinic space group $P 1$ in order to validate the assignment of the space group ( $h 00$ and $0 k 0 ; h, k=2 n+1$ reflections were unambiguously absent). Also, we solved the structure by molecular replacement in space group $P 1$ and input this solution to Zanuda (Lebedev \& Isupov, 2014). The program confirmed that space group is $P 4_{1} 2_{1} 2$.

Structure solution (at $1.7 \AA$ resolution) and refinement of coordinates (using data to $1.4 \AA$ resolution) was carried out using the PHENIX suite (Adams et al., 2010). The structure was solved by molecular replacement using a tetradecamer structure as a model (PDB entry 3v9d; Mandal et al., 2012), which was appropriately mutated to the present sequence. The top solution from phenix.phaser (McCoy et al., 2007) in terms of LLG and TFZ score was taken as an initial model and was subjected to three cycles of standard refinement using a maximum-likelihood target function with phenix.refine (Afonine et al., 2012). $5 \%$ of the data was set aside to calculate the $R_{\text {free }}$ value (Brünger, 1992). During the final refinement
Table 1

Data-processing and refinement statistics.

Values in parentheses are for the last shell.

\begin{tabular}{ll}
\hline Data processing & \\
Resolution limits $(\AA)$ & $28.06-1.40(1.43-1.40)$ \\
Space group & $P 4_{1} 2_{1} 2$ \\
Unit-cell parameters $(\AA)$ & $a=b=41.8, c=89.3$ \\
Total No. of reflections & $437650(12306)$ \\
No. of unique reflections $\dagger$ & $16170(806)$ \\
Multiplicity & $27.1(15.3)$ \\
Completeness $(\%)$ & $99.8(98.0)$ \\
Mean $I / \sigma(I)$ & $16.9(0.3)$ \\
$C_{1 / 2}(\%)$ & $100.0(29.3)$ \\
$R_{\text {merge }} \S(\%)$ & $8.1(459.6)$ \\
$R_{\text {r.i.m. }} \S(\%)$ & $8.3(475.1)$ \\
Refinement & \\
Resolution limits $(\AA)$ & $28.06-1.40(1.49-1.40)$ \\
No. of DNA atoms & 568 \\
No. of solvent atoms & 88 \\
R.m.s.d., bond lengths $(\AA)$ & 0.01 \\
R.m.s.d., bond angles $\left({ }^{\circ}\right)$ & 1.2 \\
$R_{\text {work }}(\%)$ & $22.18(36.6)$ \\
$R_{\text {free }}(\%)$ & $24.22(40.6)$ \\
Average $B$ factor $\left(\AA^{2}\right)$ & 26.26 \\
\hline
\end{tabular}

$\dagger$ Friedel pairs are considered as symmetry-equivalent reflections. $\ddagger R_{\text {merge }}=$ $\sum_{h k l} \sum_{i}\left|I_{i}(h k l)-\langle I(h k l)\rangle\right| / \sum_{h k l} \sum_{i} I_{i}(h k l)$. \& These quality indicators were not used to determine the high-resolution limit. Instead, we used $\mathrm{CC}^{*}$ and the "paired refinement' technique. - $R_{\text {r.i.m. }}=\sum_{h k l}\{N(h k l) /[N(h k l)-1]\}^{1 / 2} \sum_{i}\left|I_{i}(h k l)-\langle I(h k l)\rangle\right| \mid$ $\sum_{h k l} \sum_{i} I_{i}(h k l)$, where $N(h k l)$ is the redundancy.

cycles, the model was subjected to TLS refinement, in which chains $A$ and $B$ were treated as two separate TLS groups (Winn et al., 2001). 88 solvent molecules were identified in the electron-density map. Solvent molecules were updated automatically and the correctness of the assignment was examined at the end of each refinement cycle by inspecting the $2 F_{\mathrm{o}}-F_{\mathrm{c}}$ and $F_{\mathrm{o}}-F_{\mathrm{c}}$ electron-density maps and subsequently checking the temperature and occupancy factors. Although the crystals were grown in the presence of barium, this ion was not detected in the electron density. The final map difference showed a blob of density with a peak height of $0.3 \mathrm{e}^{-} \AA^{-3}$. This could not be interpreted as barium. The final refinement parameters are listed in Table 1. Structural analysis and geometrical calculations were carried out using w3DNA (Zheng et al., 2009). Minor-groove and major-groove widths were measured using Curves+ (Blanchet et al., 2011). PyMOL was used to generate the figures (DeLano, 2002). The atomic coordinates and structure factors have been deposited in the PDB (Berman et al., 2000) with accession code 5wv7.

\section{Results and discussion}

\subsection{Tetradecamers as A-DNA}

The present sequence d(CCGGGGTACCCCGG) ${ }_{2}$ is one of a series of tetradecamer sequences for which structures have been determined in our laboratory. These sequences were all designed with the expectation that they would form four-way junctions with different helical structures for the arms. It has been reported (Eichman et al., 2000) that a sequence with the triplet 'ACC' in its central portion would favour the formation of a four-way junction structure, and this was incorporated 
Table 2

Comparison of the average helical parameters of this structure using w3DNA (Zheng et al., 2009) with respect to the overall helical axis and fragmented helical axis with standard values for fibre A-DNA (Arnott, 1999) and A-DNA crystal structures (Olson et al., 2001).

\begin{tabular}{|c|c|c|c|c|c|c|}
\hline \multirow[b]{2}{*}{ Parameters } & \multirow[b]{2}{*}{ Overall helical axis } & \multicolumn{3}{|c|}{ Fragmented axis } & \multirow[b]{2}{*}{ Fibre A-DNA } & \multirow[b]{2}{*}{ Crystal A-DNA } \\
\hline & & Tetramer 1 & Tetramer 2 & Central hexamer & & \\
\hline Rise $(\AA)$ & $3.34(0.2)$ & $3.4(0.1)$ & $3.4(0.1)$ & $3.3(0.3)$ & 2.56 & 3.32 \\
\hline Inclination $\left({ }^{\circ}\right)$ & $6.9(10)$ & $-3.8(5.2)$ & $-0.5(1.9)$ & $14.6(6.7)$ & 22.6 & 14.7 \\
\hline Roll $\left(^{\circ}\right)$ & $4.0(5.6)$ & $-1.6(2.5)$ & $-0.2(0.9)$ & $8.6(4.2)$ & 0.0 & 8.0 \\
\hline Tilt $\left({ }^{\circ}\right)$ & $-0.5(2.8)$ & $-0.6(1.3)$ & $0.4(1.8)$ & $-1.5(3.6)$ & - & 0.1 \\
\hline Slide $(\AA)$ & $-1.7(0.5)$ & $-2.1(0.05)$ & $-2.3(0.1)$ & $-1.1(0.3)$ & -1.7 & -1.53 \\
\hline
\end{tabular}

into all of the sequences. However, the sequences d(CGCGGGTACCCGCG) $)_{2}$ (Venkadesh et al., 2011), d(CCCCGGTACCGGGG) $)_{2}$ (Mandal et al., 2012; Purushothaman et al., 2014), d(CCCGGGTACCCGGG) 2 (Karthik et al., 2017) and the present sequence all crystallize as A-type double helices, with slight variations in the structure and crystal packing. Thus, an important determinant of the structure of a DNA fragment in the crystal is apparently its length. These results may also indicate that the triplet 'GTA', which is also present in all of the sequences mentioned above, may nucleate the formation of the A-type helix, in line with the suggestion of Basham et al. (1995).

\subsection{Overall structure}

The structure of the present sequence d(CCGGGGTACCCCGG $)_{2}$ as an A-type helix is shown in Fig. 1. Table 2 gives the average helical parameters. These are close to the values for fibre A-DNA (Arnott, 1999). A uniform A-DNA fibre model for this sequence was constructed using $w 3 D N A$, with a twist value of $32.7^{\circ}$ and a rise value of $2.54 \AA$ (Arnott \& Hukins, 1972). When this model is superposed on the crystal structure, the all-atom r.m.s. deviation is $2.05 \AA$. The average value of twist for this structure is $29.7^{\circ}$ and the average rise is $3.34 \AA$. This implies a helical pitch of about $40 \AA$, with nearly 12 base pairs per turn. The fibre A-type model (Arnott, 1999) has a twist of $32.7^{\circ}$ and 11 base pairs per turn. A customized model built (Zheng et al., 2009) with the average parameters of the observed structure distributed uniformly and a fibre A-DNA model of this sequence are also shown in Fig. 2. This may be a possible extreme variant of the A-type model. In this structure, the average minor-groove width is $9.6 \AA$ and the depth is $1.4 \AA$. The values for the fibre model of A-DNA are 11.1 and $2.6 \AA$, respectively. The average major-groove width is $6.2 \AA$ and the depth is $9.3 \AA$. The values are 2.2 and $13 \AA$, respectively, for the fibre model (Arnott, 1999).

The crystal structure is not uniform, but shows a large amount of variation at each base step (Supplementary Table S2, Supplementary Fig. S2). The variation does not appear to be correlated with the sequence: when one half of the helix is superposed on the other half of the helix, the relatively high r.m.s.d. value of $0.73 \AA$ suggests that the two halves are structurally different, even though the sequence is symmetrical. This point is also made in Fig. 3, where the parameters are not symmetrically distributed in the two halves of the helix.

\subsection{Choice of fragment axis}

The helix has an overall bend of $19^{\circ}$. The degree of bending at the central hexamer is distinct from the bend at the terminal tetramers. Thus, the molecule can be considered as being composed of three short helical fragments (Fig. 4). Table 2 gives a comparison of the helical parameters corresponding to
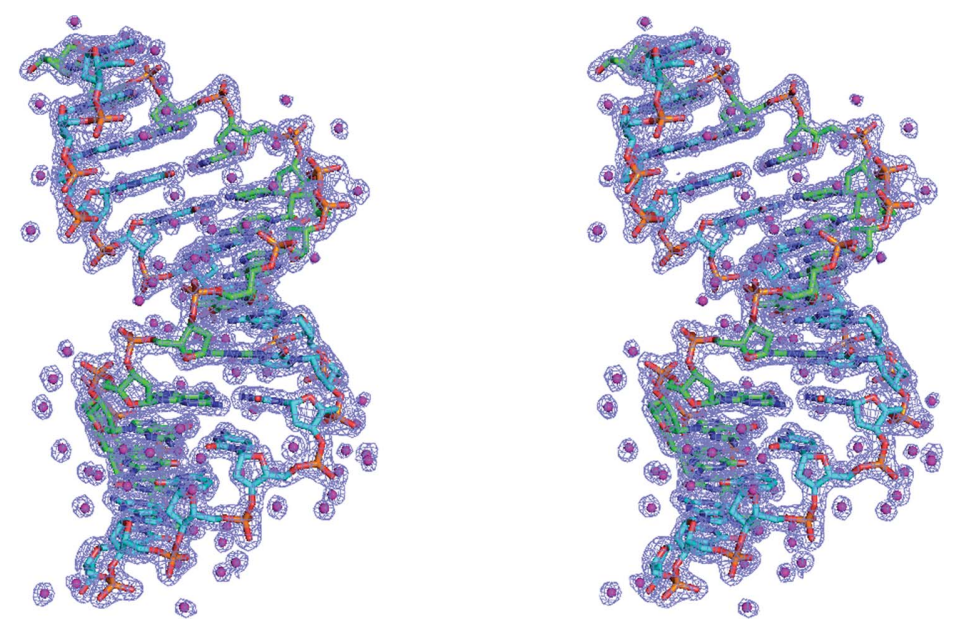

Figure 1

Stereoview of the DNA and solvent molecules in the asymmetric unit: $2 F_{\mathrm{o}}-F_{\mathrm{c}}$ map contoured at the $1 \sigma$ level. 
the overall helical axis and to the three fragmented axes. It is clear that the tetramers at the two ends of the sequence have a significantly different structure from the hexamer in the middle. The individual values of the rise are notably larger, and the values of twist notably smaller, at the ends than in the middle, where they are much closer to the average values for A-type crystal structures (Olson et al., 2001). Another striking difference is seen in the values of inclination. One of the notable features of the A-type helical model, especially when compared with the B-type model, is the large inclination of the bases from the 'horizontal', i.e. the plane perpendicular to the helical axis. This value is $22.6^{\circ}$ in the fibre model. In the crystal structures this parameter has an average value of $14.7^{\circ}$. At the two ends of the present sequence the inclination is small or even negative, while in the central hexamer it is large and positive. The implication of this is that the two ends of the helix have a structure that is quite different from the 'canonical' A-type helix, while the central hexamer can be more properly characterized as the A-form. It has been observed (Wahl \& Sundaralingam, 1997) that for longer ADNA helices crystal-packing forces exert a larger effect than the base sequence on the conformational parameters. It has also been observed (Shakked \& Rabinovich, 1986) that variations from the fibre model occur more frequently at the



(a)

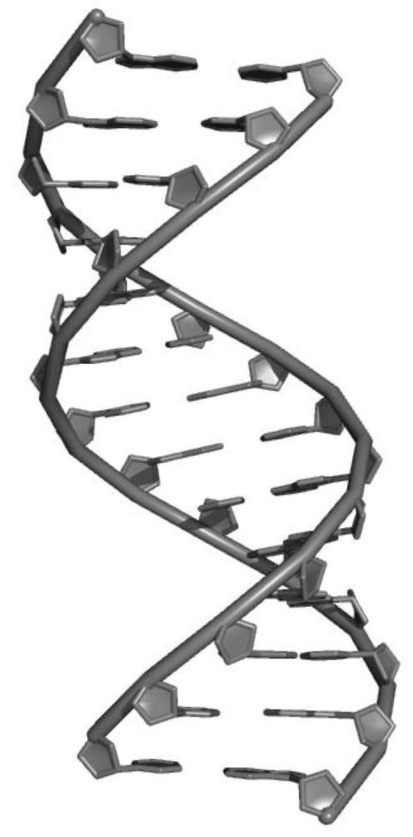

(b)

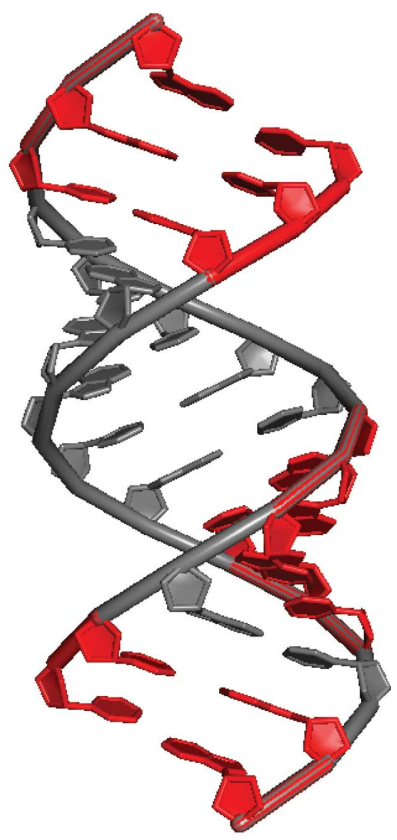

(c)

Figure 2

View (perpendicular to the helix axis) of $(a)$ the present structure, $(b)$ a customized model and $(c)$ fibre A-DNA. The base-pair steps with distinct structural parameters are represented in red.
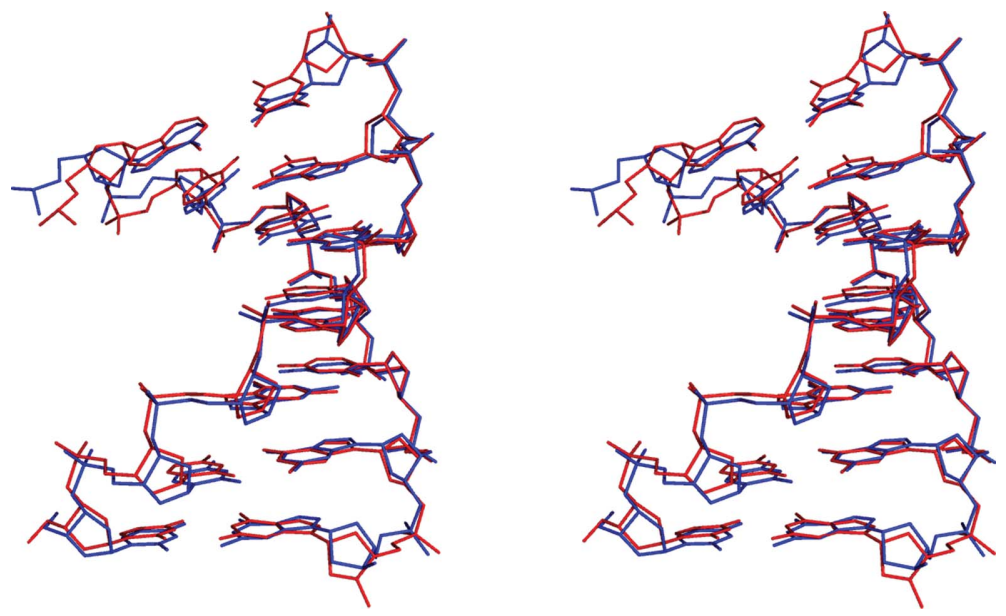

Figure 3

Stereoview of the superposition of one heptamer half of the duplex on the other. 
ends of the helix. In the present structure also, the bending of the helix and the structural variations observed may be owing to crystal-packing forces.

\subsection{Crystal packing}

The sequence $\mathrm{d}\left(\mathrm{CCCCGGTACCGGGG)}\right.$ (which was used $_{2}$ as a model in the molecular-replacement calculations for the solution of the present structure) is also a tetradecamer that crystallizes as A-type DNA (Mandal et al., 2012). Its crystals belong to space group $P 4_{1}$, while the present sequence crystallizes in space group $P 4_{1} 2_{1} 2$. Although both crystals belong to the tetragonal system, the $a$ and $b$ unit-cell parameters of the previously reported structure are $1 /\left(2^{1 / 2}\right)$ of those of the present structure. A comparison of the smaller and the larger unit cells shows that the latter is formed by four of the former. The change in space group between the two structures could be owing to the presence of a well defined manganese ion in the previously reported structure, whereas in the present structure we could not identify any metal ions in the electrondensity map, although the crystallization condition contained barium chloride. In order to reaffirm the presence of higher symmetry in the observed unit-cell parameters, we reindexed the frames in lower symmetric space groups such as $P 2_{1} 2_{1} 2_{1}$ and $P 4_{1}$. The redundancy-independent merging $R$ factor $\left(R_{\text {r.i.m. }}\right)$ is almost exactly the same in the different space groups

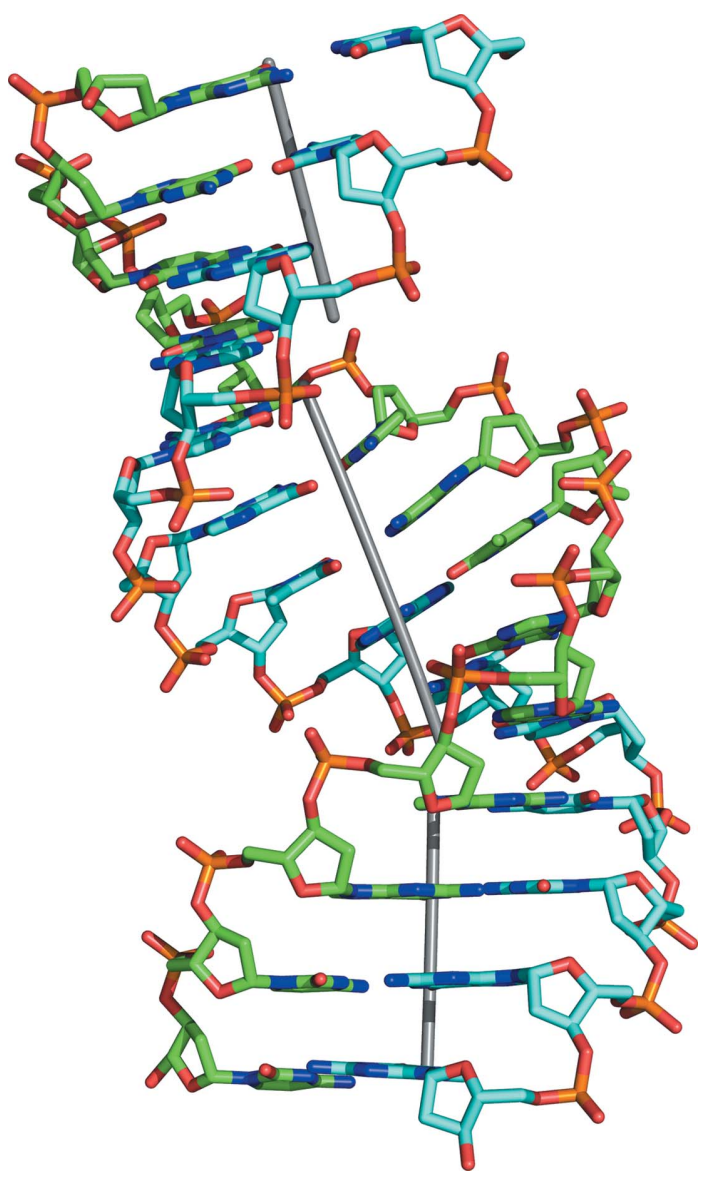

Figure 4

Illustration of the choice of three fragment axes rather than the choice of global axes.
(Fig. 5). Attempts to index and integrate the frames in the smaller unit cell $(a=b=29, c=89 \AA)$, which had identical figures of merit as the larger unit cell, failed. The major interhelical interaction observed was stacking between the terminal base pair and flat minor-groove sugars of a neighbouring helix (Fig. 6a). This kind of interaction is present in all A-DNA crystal structures (Wahl \& Sundaralingam, 1997). In contrast to A-DNA, the duplexes of B-DNA and Z-DNA stack coaxially on top of each other, leaving the grooves free from interactions with neighbouring molecules. Essentially, the hydration shell in this portion of the minor groove of A-DNA is completely displaced by the hydrophobic surface of the base pairs. This is consistent with the fact that A-DNA is favoured in an environment of reduced water activity. Other interhelical interactions that are observed in the structure are the following: (i) between the minor-groove atoms of one helix and the minor-groove atoms of a symmetry-related neighbour (Fig. 6b), (ii) between the minor-groove atoms of one helix and the backbone atoms of a symmetry-related neighbour (Fig. 6c), and (iii) between the backbone atoms of two helices (Fig. $6 d$ ), a type of interaction that we have previously categorized as a backbone-backbone type 2 interaction (Venkadesh et al., 2011).

\subsection{Hydration}

Water is an integral part of the nucleic acid structure. It occupies $60 \%$ of the present unit-cell content. We were able to identify 88 water molecules in the electron-density map. Of the water molecules observed, 30 are in the major groove of the helix, 24 occupy sites on the nearly flat minor-groove surface or at the termini, and 34 are coordinated to the phosphate groups. Of this last group, five waters are involved in bridging between the phosphate groups, while the other 29 hydrate the phosphates separately.

The relationship between DNA conformation and hydration has been described previously. B-type DNA has a characteristic 'spine of hydration' (Drew \& Dickerson, 1981) running along the minor groove. In the case of A-DNA, the 'economics of hydration' (Saenger et al., 1986) favour water



Figure 5

$R_{\text {r.i.m. }}$ versus $\sin (\theta) / \lambda$ plotted for the assigned space group and the two maximal subgroups. 


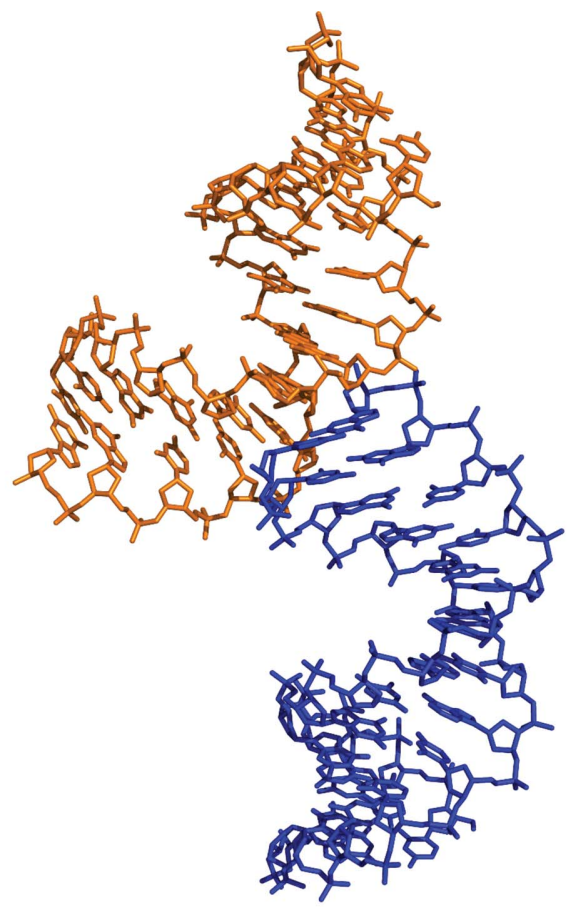

(a)



(c)



(b)

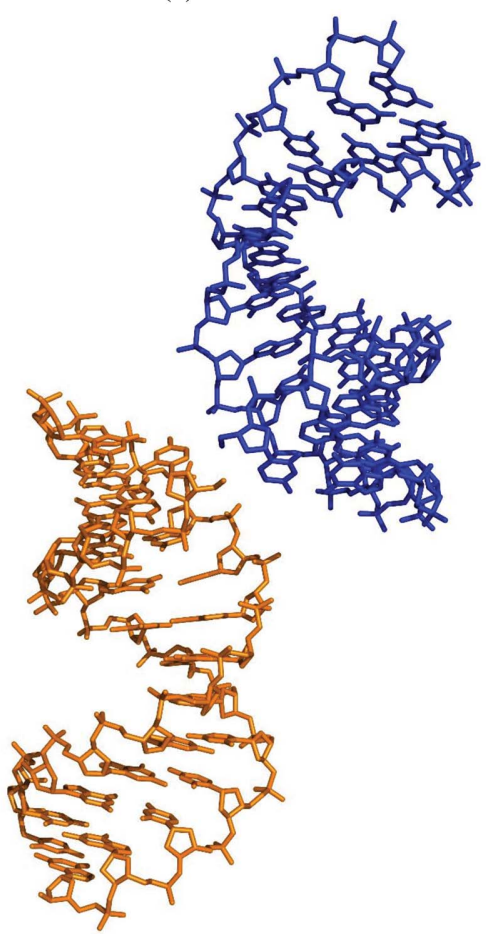

(d)

Figure 6

Packing motifs. (a) Terminus-minor groove interaction. (b) Minor groove-minor groove interaction. (c) Minor groove-backbone interaction. (d) Backbone-backbone interaction.

molecules surrounding the phosphate groups rather than attaching themselves to the atoms of the bases.

\section{Conclusion}

We have solved the structure of the tetradecamer oligonucleotide sequence d(CCGGGGTACCCCGG) $)_{2}$ at a resolu- tion of $1.4 \AA$. The crystallographic asymmetric unit consists of a right-handed A-type double helix. The average helical parameters twist and rise are significantly different from those seen in the fibre model. The helix may thus be characterized as a variant of the A-type helix. The helix is bent at two places along the sequence, and the helix is better understood as a tetramer joined to a hexamer, which is again joined to a 
tetramer. Although these variations may be a consequence of crystal-packing forces rather than the sequence, these forces are the same as in other A-type DNA crystal structures, where such structural variations are not observed.

\section{Acknowledgements}

We acknowledge the Department of Biotechnology, Government of India for X-ray data collection at BM14, ESRF, France. We also thank UGC for grants under the CAS program. SK acknowledges the DST for an INSPIRE fellowship and AT acknowledges UGC for a UGC-BSR fellowship.

\section{References}

Adams, P. D. et al. (2010). Acta Cryst. D66, 213-221.

Afonine, P. V., Grosse-Kunstleve, R. W., Echols, N., Headd, J. J., Moriarty, N. W., Mustyakimov, M., Terwilliger, T. C., Urzhumtsev, A., Zwart, P. H. \& Adams, P. D. (2012). Acta Cryst. D68, 352-367.

Arnott, S. (1999). Oxford Handbook of Nucleic Acid Structure, edited by S. Neidle, pp. 1-38. Oxford University Press.

Arnott, S. \& Hukins, D. W. (1972). Biochem. Biophys. Res. Commun. 47, 1504-1509.

Basham, B., Schroth, G. P. \& Ho, P. S. (1995). Proc. Natl Acad. Sci. USA, 92, 6464-6468.

Berman, H. M., Westbrook, J., Feng, Z., Gilliland, G., Bhat, T. N., Weissig, H., Shindyalov, I. N. \& Bourne, P. E. (2000). Nucleic Acids Res. 28, 235-242.

Blanchet, C., Pasi, M., Zakrzewska, K. \& Lavery, R. (2011). Nucleic Acids Res. 39, W68-W73.

Brünger, A. T. (1992). Nature (London), 355, 472-475.
DeLano, W. L. (2002). PyMOL. http://www.pymol.org.

Drew, H. R. \& Dickerson, R. E. (1981). J. Mol. Biol. 151, 535-556.

Eichman, B. F., Vargason, J. M., Mooers, B. H. \& Ho, P. S. (2000). Proc. Natl Acad. Sci. USA, 97, 3971-3976.

Kabsch, W. (2010). Acta Cryst. D66, 125-132.

Karplus, P. A. \& Diederichs, K. (2012). Science, 336, 1030-1033.

Karthik, S., Thirugnanasambandam, A., Mandal, P. K. \& Gautham, N. (2017). Nucleosides Nucleotides Nucleic Acids, https://doi.org/ 10.1080/15257770.2017.1287378.

Lebedev, A. A. \& Isupov, M. N. (2014). Acta Cryst. D70, 2430-2443.

Mandal, P. K., Venkadesh, S. \& Gautham, N. (2012). Acta Cryst. F68, 393-399.

Matthews, B. W. (1968). J. Mol. Biol. 33, 491-497.

McCoy, A. J., Grosse-Kunstleve, R. W., Adams, P. D., Winn, M. D., Storoni, L. C. \& Read, R. J. (2007). J. Appl. Cryst. 40, 658-674.

Olson, W. K. et al. (2001). J. Mol. Biol. 313, 229-237.

Padilla, J. E. \& Yeates, T. O. (2003). Acta Cryst. D59, 1124-1130.

Purushothaman, M., Varghese, A., Mandal, P. K. \& Gautham, N. (2014). Acta Cryst. F70, 860-865.

Rossmann, M. G., Blow, D. M., Harding, M. M. \& Coller, E. (1964). Acta Cryst. 17, 338-342.

Saenger, W., Hunter, W. N. \& Kennard, O. (1986). Nature (London), 324, 385-388.

Shakked, Z. \& Rabinovich, D. (1986). Prog. Biophys. Mol. Biol. 47, 159-195.

Venkadesh, S., Mandal, P. K. \& Gautham, N. (2011). Biochem. Biophys. Res. Commun. 407, 307-312.

Wahl, M. C. \& Sundaralingam, M. (1997). Biopolymers, 44, 45-63.

Winn, M. D., Isupov, M. N. \& Murshudov, G. N. (2001). Acta Cryst. D57, 122-133.

Zheng, G., Lu, X.-J. \& Olson, W. K. (2009). Nucleic Acids Res. 37, W240-W246.

Zwart, P. H., Grosse-Kunstleve, R. W. \& Adams, P. D. (2005). CCP4 Newsl. Protein Crystallogr. 43, contribution 7. 